Edit Bors, Pázmány Péter Catholic University, Hungary

DOI: $10.17951 /$ Ismll.2020.44.1.105-112

\title{
La langue secrète du visage : représentations des émotions dans les tragédies de Racine
}

\author{
The Secret Language of the Face : Representations of Emotions \\ in Racine's Tragedies
}

\begin{abstract}
RÉSUMÉ
Dans son essai L'effet de sourdine dans le style classique: Racine, Léo Spitzer étudie les procédés d'atténuation plus particulièrement ceux relatifs au visage (regard, yeux, bouche). Comment aborder la notion du visage ? Et comment la mettre à profit dans l'analyse du langage du théâtre classique ? Nous postulons que le visage, étant au service de l'expression des sentiments profonds, est en étroite relation avec le corps même et avec ce «bouillonnement intérieur » qui anime les personnages raciniens tout en les poussant à se cacher derrière un masque anonyme, officiel ou collectif.

Mots-clés : visage, émotions, atténuation, masque, Racine
\end{abstract}

\section{ABSTRACT}

In his essay L'effet de sourdine dans le style classique: Racine, Léo Spitzer analyzes attenuation mechanisms especially in connection with the face (gaze, eyes, mouth). How to approach the idea of face ? And how to use it in the analysis of classical theater's language? We presume that the face systematically expresses deep emotions and is closely connected to the body and to this « bouillonnement intérieur » (inner excitement) that agitates the Racinian heroes by Racine's tragedies. Keywords: face, emotions, attenuation, mask, Racine

\section{Introduction}

Léo Spitzer demeure, en matière de stylistique, une référence principale. Comme le dit Starobinski, « pour caractériser des traits de style, il sait créer des notions neuves, gagnées aux prix de l'attention la plus exquise : il invente des expressions frappantes et justes telles que « atténuation classique » ou « effet de sourdine » [klassische Dämpfung] » (Spitzer, 1980, p. 19). Dans son essai, qui porte le titre L'effet de sourdine dans le style classique : Racine (Spitzer, 1980, pp. 208-336), il étudie les occurrences des procédés d'atténuation dont nous ne repérerons que ceux relatifs au visage des personnages : leurs faces visibles (regard, yeux,

Edit Bors, Pázmány Péter Katolikus Egyetem - Bölcsészet- és Társadalomtudományi Kar, Klasszikus és Újlatin Nyelvek Intézete - Francia Tanszék, Bertalan Lajos utca 2, 1111 Budapest, bors.edit@btk.ppke.hu, https://orcid.org/0000-0001-6873-462 
bouche, etc.) et leurs masques invisibles étant au service de l'expression directe ou indirecte de sentiments profonds.

Comment aborder la notion du visage ? Et comment la mettre à profit dans l'analyse du théâtre classique ? En effet, le visage se trouve en relation étroite avec la relation à l'autre (Salmon, 2012, p. 104) :

Ce visage entrevu de l'autre ne serait-il pas la marque du désir ? S'il est vrai que tout désir se porte vers ce qui est autre, vers ce qui n'est pas accessible d'emblée, vers ce qui manque, le visage n'est-il pas ce que l'on ne peut atteindre, alors même qu'il est présent, bien réel, inscrit dans le monde comme moi ?

Le visage de l'autre, comme la marque du désir et de la passion, est bien présent dans les tragédies de Racine, notamment, dans Bérénice, Phèdre et Andromaque : il suffit de citer ici les remarques de Spitzer concernant le pouvoir des yeux qui permettent " l'expression certes la plus intellectuelle du sentiment amoureux » (Spitzer, 1980, p. 303). La fonction des yeux est, comme il le dit lui-même (Spitzer, 1980, p. 305), assez variée : d'une part, les yeux sont vus concrètement dans les gestes, tout en étant aussi le miroir de l'âme, d'autre part, ils sont considérés comme des êtres indépendants qui agissent ou comme de simples formules pour remplacer je, tu ou il.

Toutefois, le visage ne se limite pas à la face : « Le visage n'existe pas en soi, il n'est pas à proprement parler visible, mais il est dans une face $[\ldots] »$, dit Drevet (2003, p. 21). Plus loin, il précise la même idée (p. 22) : «Visage : l'invisible de la face mais qui l'anime» ou «Le visage est question moins de traits que de gestes, moins de configuration que de façon, moins de symétrie que de port» (p. 23).

Cette conception élargie du visage a ceci d'avantageux qu'il nous permettra de prendre en considération aussi bien la face que le corps même, l'allure et le port, et ce « bouillonnement intérieur» (Spitzer, 1980, p. 210) qui anime les personnages raciniens tout en les poussant à se cacher derrière un masque anonyme, officiel ou collectif.

\section{Les figures de la face visible et les troubles du langage}

Les passions raciniennes correspondent, pour Barthes (2002), à une sorte d'aliénation due à un " amour immédiat ", qui naît brusquement et s'empare du héros ou de l'héroïne surtout par la vue. L'aliénation se manifeste dans le désordre charnel et les troubles physiques (rougeur, pâleur, soupirs, pleurs), comme l'observe Barthes. Dans les exemples suivants

(1)

\section{PHÈDRE}

Je le vis, je rougis, je pâlis à sa vue ; / Un trouble s'éleva dans mon âme éperdue ; / Mes yeux ne voyaient plus, je ne pouvais parler ; / Je sentis tout mon cœur et transir et brûler (Racine, 1971b, p. 48). 
(2)

PHÈDRE

Insensée, où suis-je ? et qu'ai-je dit ? / Où laissé-je égarer mes vœux et mon esprit ? / Je l'ai perdu : les Dieux m'en ont ravi l'usage. / Enone, la rougeur me couvre le visage : / Je te laisse trop voir mes honteuses douleurs ; / Et mes yeux, malgré moi, se remplissent de pleurs (Racine, 1971b, p. 44).

on voit bien que le personnage est saisi surtout par la vue et se sent agi en même temps par une force extérieure (les Dieux m'en ont ravi l'usage). Cette division du moi s'observe de préférence dans les monologues (Barthes, 2002) ou éventuellement dans les entretiens avec les confident(e)s. Outre les signes de la face visible, le désordre prend forme dans l'impossibilité ou l'interdiction de la parole (Barthes, 2002). Le mutisme est source de tragédie dans le cas de Titus qui se fait aphasique pour rompre avec Bérénice. Comme dans (3) et (4) :

(3)

BÉRÉNICE

Hé bien, Seigneur ? Mais quoi ! sans me répondre / Vous détournez les yeux, et semblez vous confondre. / Ne m'offrirez-vous plus qu'un visage interdit ? / Toujours la mort d'un père occupe votre esprit? (Racine, 1971a, p. 61).

(4)

Titus

Ma bouche et mes regards, muets depuis huit jours, / L'auront pu préparer à ce triste discours : / Et même en ce moment, inquiète, empressée, / Elle veut qu'à ses yeux j'explique ma pensée (Racine 1971a, p. 69).

Les paroles de Bérénice, tout en faisant preuve de fortes émotions (exclamations, interrogations), et tout en reflétant les signaux non verbaux (détourner les yeux, visage interdit) de l'embarras de Titus, prennent la forme d'une plainte, l'une des techniques d'agression verbale dont parle Barthes (2002). La plainte est aussi « l'expression d'une souffrance dans la relation à l'autre » (de Butler, 2004, p. 58) ; pour Freud, la plainte serait « un moyen de pallier cette souffrance, de provoquer le retour de l'objet en le séduisant et de se l'attacher » (de Butler, 2004, p. 59). En effet, Bérénice, bien que souffrante, au lieu de faire des reproches, se plaint de l'absence de Titus en essayant de le forcer à retrouver un langage. La violence du langage se manifeste plus librement dans la stichomythie, qui est pour Spitzer « un duel oratoire, où les lames des combattants s'entrechoquent avec éclat» (Spitzer, 1980, p. 264). Plus précisément, comme le dit Ryngaert (1995, p. 89),

[...] la stichomythie fait alterner des répliques brèves (un vers ou quelques vers, parfois deux ou trois mots), en principe de même longueur, qui font penser à un duel verbal quand monte la tension dramatique. 
Spitzer (1980, p. 279) décrit divers «procédés techniques pour dépeindre l'égarement ", pour dévoiler le vrai visage des personnages, entre autres la répétition solennelle à l'aide de laquelle « les sentiments des personnages semblent s'épancher sans entrave ». Dans l'exemple (5),

(5)

THÉRAMĖNE

Excusez ma douleur. Cette image cruelle / Sera pour moi de pleurs une source éternelle. / J'ai vue, Seigneur, j'ai vu votre malheureux fils / Traîné par les chevaux que sa main a nourris. / Il veut les rappeler, et sa voix les effraie. / Ils courent. Tout son corps n'est bientôt qu'une plaie (Racine, 1971b, pp. 111-112).

Théramène, le gouverneur d'Hippolyte, raconte à Thésée, sous le coup d'émotions fortes, la mort de son fils. L'émotivité du témoignage est due notamment à la répétition du verbe j'ai vu, et à la présence des déterminants possessifs. Acela s'ajoute l'emploi du présent historique correspondant à l’hypotypose, figure rhétorique qui

peint les choses d'une manière si vive et si énergique, qu'elle les met en quelque sorte sous les yeux et fait d'un récit ou d'une description, une image, un tableau, ou même une scène vivante (Herschberg-Pierrot, 1999, p. 79).

En revanche, les phrases comme « Muet, chargé de soins, et les larmes aux yeux » (Bérénice) illustrent l'asyndète de gradation grâce à laquelle on " sent les ondes successives d'une douce tristesse, [...] qui s'enfoncent de plus en plus avant dans le cœur » (Spitzer, 1980, p. 282). D'autres cas de gradation correspondent plutôt à un « crescendo stylistique » (Spitzer, 1980, p. 282) qui reflète le désarroi du personnage. Comme dans l'exemple (6) reproduisant les paroles d'Oreste à qui Hermione demande de tuer Pyrrhus :

(6)

ORESTE

A peine suis-je encore arrivé dans l'Épire, / Vous voulez par mes mains renverser un empire ; / Vous voulez qu'un roi meure ; et pour son châtiment / Vous ne donnez qu'un jour, qu'une heure, qu'un moment ; / [...] (Racine, 1965, p. 95).

Et pour terminer cette première partie, citons à titre d'exemple l'exclamation et l'interjection (voir (3) et (7))

(7)

BÉRÉNICE

Nous séparer? Qui ? Moi ? Titus de Bérénice! (Racine, 1971a, p. 77).

qui sont l'expression d'un sentiment vif et immédiat, comme le dit Spitzer. Avec l'interjection, continue-t-il, « les maximes impersonnelles sont baignées de la 
chaleur du sentiment individuel » (Spitzer, 1980, p. 294), les ah, hélas, quoi sont " des cris de douleur de la nature humaine condamnée sans espoir à la souffrance » (Spitzer, 1980, p. 294).

\section{Les figures du « bouillonnement intérieur »}

Dans le discours racinien, cependant, l'égarement (Spitzer) ou l'aliénation (Barthes) ont tendance à se masquer grâce aux différents effets d'atténuation décrits et analysés en profondeur par Spitzer.

C'est le cas par exemple du procédé de désindividualisation par l'article indéfini grâce auquel « le cas individuel est ignoré, tout est dit sous le rapport du général » (Spitzer, 1980, p. 210). L'emploi de l'article indéfini crée une certaine distance (Spitzer, 1980, p. 210) impersonnelle et apparaît lorsque le moi cherche à se voiler sans renoncer à ses droits (Spitzer, 1980, p. 211). Malgré cette distance, les sentiments sont « profondément enfouis » et « nous pressentons un bouillonnement intérieur », dit Spitzer (1980, p. 210). Comme dans (8) :

(8)

ORESTE

Assez et trop longtemps mon amitié t'accable : / Évite un malheureux, abandonne un coupable (Racine, 1965, p. 72).

Dans son entretien avec son ami Pylade, Oreste, au lieu d'adopter un ton subjectif, se nomme un malheureux, un coupable pour tenter de se réfugier dans une généralité désindividualisée, derrière un masque anonyme. A cela s'oppose le mouvement contraire de la gradation (Évite un malheureux, abandonne un coupable) qui reproduit en même temps ce « bouillonnement intérieur » des sentiments étouffés.

Le même effet se produit lors de l'utilisation du nom propre (voir (7)) ou du pluriel de majesté (voir (9)) qui cachent imperceptiblement le personnage derrière un masque officiel. On voit bien que noblesse oblige : le personnage se place au niveau du fait général pour retrouver la grandeur. En effet, comme le dit Spitzer (1980) :

Le nom est en quelque sorte l'impératif catégorique du personnage - mais cet impératif moral a quelque chose de didactique, de réflexif, d'atténuant; il tue le lyrisme (p. 222).

Le pluriel de majesté, autre facteur de la dépersonnalisation du discours (Spitzer, 1980, p. 225), alterné avec la première personne crée le même effet : tantôt le Moi se découvre, tantôt il se cache derrière un masque collectif. Comme on peut l'observer à partir du monologue d'Antiochus : 
(9)

ANTIOCHUS

Après cinq ans d'amour et d'espoirs superflus, / Je pars, fidèle encor quand je n'espère plus. / $\mathrm{Au}$ lieu de s'offenser, elle pourra me plaindre. / Quoi qu'il en soit, parlons ; c'est assez nous contraindre, / Et que peut craindre, hélas ! un amant sans espoir / Qui peut bien se résoudre à ne la jamais voir? (Racine, 1971a, p. 34).

Dans cet extrait, le pluriel de majesté n'est pas le seul procédé d'atténuation, il est aussi renforcé par le procédé de désindividualisation par l'article indéfini (un amant sans espoir). Toutefois, derrière le masque anonyme jaillit la souffrance (voir aussi l'interjection hélas!) de l'individu dont le désir secret est d'être aimé ou au moins de susciter la pitié.

L'atténuation de l'expression directe du sentiment s'observe aussi dans l'emploi du démonstratif de distance. L'emploi du démonstratif, dit Spitzer, " supprime la chaleur humaine du possessif » (Spitzer, 1980, p 215) ou encore « le possessif dénoterait une certaine participation, - le démonstratif se cantonne dans une neutralité froide et circonspecte » (Spitzer, 1980, p. 216) Comme dans (10):

(10)

BÉRÉNICE

Hé bien ! régnez cruel ; contentez votre gloire : / je ne dispute plus. J'attendais, pour vous croire, / Que cette même bouche, après mille serments / D'un amour qui devait unir tous nos moments / Cette bouche, à mes yeux s'avouant infidèle, / M'ordonnât elle-même une absence éternelle. / Moi-même, j'ai voulu vous entendre en ce lieu. / Je n'écoute plus rien, et pour jamais adieu (Racine, 1971a, p. 87).

Dans cet exemple, les termes relatifs au visage (bouche) en tant qu'anaphores associatives (Gardes-Tamine \& Pelizza, 1998, p. 153) impliquent des relations lexicales particulières, comme celle de partie de corps à la totalité de l'individu. Toutefois, l'emploi du démonstratif associé à des parties du corps (cette bouche) signale que l'individu « se devient lui-même étranger» (Spitzer, 1980, p. 216), tout comme les tournures en ce lieu pour 'ici' qui renferment une certaine idée de distance pour indiquer que le personnage apparaît étranger au lieu aussi (Spitzer, 1980, p. 217).

Le procédé de la personnification des abstraits permet le passage de l'individuel à l'universel. Racine fait agir ainsi non pas des personnages, mais des forces abstraites qui meuvent ces personnages (Spitzer, 1980, p. 227). Dans Andromaque,

HERMione

Où suis-je ? Qu'ai-je fait? Que dois-je faire encore ? / Quel transport me saisit ? Quel chagrin me dévore? (Racine, 1965, p. 103). 
les forces abstraites (transport, chagrin) s'emparent de l'héroïne et fonctionnent en tant que masque cachant un personnage en proie à une jalousie féroce.

A la fin de cette deuxième partie, il convient de souligner que les procédés d'atténuation dont nous avons parlé, se combinent de préférence pour renforcer le contraste entre l'ardeur des sentiments et l'impersonnalité du ton adopté, comme dans (12):

\section{(12)}

\section{PHĖDRE}

J'ai langui, j'ai séché, dans les feux, dans les larmes. / Il suffit de tes yeux pour t'en persuader, / Si tes yeux un moment pouvaient me regarder. / Que dis-je ? Cet aveu que je viens de faire, / Cet aveu si honteux, le crois-tu volontaire? (Racine, 1971b, p. 68).

Cet exemple pris dans l'aveu de Phèdre réunit plusieurs procédés de distanciation : l'emploi métonymique des parties du corps (yeux) remplaçant le personnage ; les forces abstraites, étant cette fois-ci au pluriel (les feux) pour estomper les contours (Spitzer, 1980, p. 230) et pour empêcher une détermination trop nette de l'attitude du personnage (Spitzer, 1980, p. 231) ; le démonstratif de distance (cet aveu) qui, en se substituant au possessif, a pour fonction d'atténuer la force des propos et des sentiments; et le « si d'affirmation forte » (si honteux) qui, comme le dit Spitzer (1980, p. 219) marque un refroidissement, un affaiblissement dus à la fréquence de l'usage.

\section{Conclusion}

La relecture de l'essai de Léo Spitzer nous a permis d'attirer l'attention sur la dynamique que produisent l'opposition de la face visible et du « bouillonnement intérieur » derrière les masques anonyme, officiel et collectif. Ces mouvements contraires s'illustrent par le bel exemple des paroles d'Oreste :

\section{(13) \\ ORESTE}

Et vous le haïssez? Avouez-le, madame, / L'amour n'est pas un feu qu'on renferme en une âme :

/ Tout nous trahit, la voix, le silence, les yeux ; / Et les feux mal couverts n'en éclatent que mieux (Racine, 1965, p. 61).

Comment les personnages essaient-ils donc de couvrir les feux ? Par la grande variété des procédés d'atténuation, tels que le nom propre, le pluriel de majesté ou la personnification des abstraits. D'un côté, ces exemples montrent clairement, comme le dit aussi Spitzer, " pourquoi nous ressentons chez Racine, en dépit du lyrisme et de la profondeur psychologique, quelque chose d'un peu froid, une distance, une sourdine » (Spitzer, 1980, p. 308), mais aussi un bouillonnement intérieur des sentiments venus du fond du cœur pour constituer les fondements 
d'un vrai visage. De l'autre côté, les personnages raciniens font souvent preuve d'égarement ou d'aliénation dont les symptômes, le désordre de la face visible et les troubles du langage, semblent contrarier le pouvoir des masques qui non seulement cachent mais révèlent des passions secrètes.

\section{References}

Barthes, R. (2002). Sur Racine. Paris: Seuil.

De Butler, A. (2004). Plainte et séduction. Dialogue, 2, 58-71. DOI: 10.3917/dia.164.0058.

Drevet, P. (2003). Le désir du visage. Les cahiers de médiologie, 15, 17-23. DOI: 10.3917/ cdm.015.0017.

Gardes-Tamine, J., \& Pelizza, M. A. (1998). La construction du texte. De la grammaire au style. Paris: Armand Colin.

Herschberg-Pierrot, A. (1999). Stylistique de la prose. Paris: Belin.

Racine, J. B. (1965). Andromaque, Paris: Librairie Larousse.

Racine, J. B. (1971a). Bérénice. Paris: Librairie Larousse.

Racine, J. B. (1971b). Phèdre. Paris: Librairie Larousse.

Ryngaert, J.-P. (1995). Introduction à l'analyse du théâtre. Paris: Dunod.

Salmon, M. (2012). La trace dans le visage de l'autre. Sens-dessous, 10, 102-111. DOI: 10.3917/ sdes.010.0102.

Spitzer, L. (1980). Études de style. Paris: Gallimard. 